\title{
Nilotinib: optimal therapy for patients with chronic myeloid leukemia and resistance or intolerance to imatinib
}

\author{
Ronan Swords \\ Devalingam Mahalingam \\ Swaminathan Padmanabhan \\ Jennifer Carew \\ Francis Giles \\ Institute for Drug Development, \\ Cancer Therapy and Research Centre, \\ University of Texas Health Science \\ Centre at San Antonio, USA
}

\begin{abstract}
Chronic myeloid leukemia (CML) is the consequence of a single balanced translocation that produces the BCR-ABL fusion oncogene which is detectable in over $90 \%$ of patients at presentation. The BCR-ABL inhibitor imatinib mesylate (IM) has improved survival in all phases of $\mathrm{CML}$ and is the standard of care for newly diagnosed patients in chronic phase. Despite the very significant therapeutic benefits of IM, a small minority of patients with early stage disease do not benefit optimally while IM therapy in patients with advanced disease is of modest benefit in many. Diverse mechanisms may be responsible for IM failures, with point mutations within the Bcr-Abl kinase domain being amongst the most common resistance mechanisms described in patients with advanced CML. The development of novel agents designed to overcome IM resistance, while still primarily targeted on BCR-ABL, led to the creation of the high affinity aminopyrimidine inhibitor, nilotinib. Nilotinib is much more potent as a BCR-ABL inhibitor than IM and inhibits both wild type and IM-resistant BCR-ABL with significant clinical activity across the entire spectrum of BCR-ABL mutants with the exception of T315I. The selection of a second generation tyrosine kinase inhibitor to rescue patients with imatinib failure will be based on several factors including age, co-morbid medical problems and ABL kinase mutational profile. It should be noted that while the use of targeted BCR-ABL kinase inhibitors in CML represents a paradigm shift in CML management these agents are not likely to have activity against the quiescent CML stem cell pool. The purpose of this review is to summarize the pre-clinical and clinical data on nilotinib in patients with CML who have failed prior therapy with IM or dasatinib.
\end{abstract}

Keywords: nilotinib, chronic myeloid leukemia, imatinib

\section{Introduction}

Chronic myeloid leukemia (CML) is a clonal myeloproliferative disorder characterized by the presence of the Philadelphia chromosome $(\mathrm{Ph})$, which is the result of a balanced reciprocal translocation involving the long arms of chromosomes 9 and 22. The fusion gene that emerges (BCR-ABL) encodes a constitutively active protein tyrosine kinase that is primarily responsible for the leukemic phenotype. ${ }^{1,2}$ The disease typically affects patients with a median age of 66 years and accounts for $7 \%$ to $15 \%$ of newly diagnosed leukemia in adults. The incidence of CML is approximately 1 to 1.5 cases per 100,000 population in the United States. ${ }^{3}$ According to the National Cancer Institute SEER (Surveillance and Epidemiology End Results) database, close to 4830 new cases will be diagnosed in the US in 2008 and of these, 450 patients will succumb to the disease. The natural history of CML begins with a chronic indolent phase (CML-CP) followed by an accelerated phase (CML-AP) and finally a blastic phase (BP) with transition being usually to either acute myeloid or acute lymphoblastic leukemia (AML, ALL). In the early days of CML management, treatment relied heavily on agents like hydroxyurea and busulfan with rapid progression to blastic phase disease and early death. Outcomes for CML patients 
improved somewhat with the arrival of cytarabine (Ara-C) and interferon (IFN); however, the prospect for long-term disease free survival was limited given the lack of efficacy of available agents and the treatment related mortality (TRM) associated with allogeneic stem cell transplantation. The prognosis of patients with CML improved dramatically in the late 1990s following the introduction of imatinib mesylate (IM, Gleevec ${ }^{\circledR}$, Glivec $^{\circledR}$; Novartis Pharmaceuticals), a first in class novel and selective inhibitor of Bcr-Abl. In newly diagnosed patients with CML, IM is now associated with complete response rates of $87 \%$, progression rates to advanced disease (accelerated and blastic phase) of 7\% and an estimated 5-year survival rate of $89 \%$, over $95 \%$ if confined to CML-related causes of death. ${ }^{46}$ The development of IM represents one of the most significant advances thus far achieved in anti-cancer therapy. While the introduction of IM has revolutionized the management of CML, there is a small minority of patients who will display primary or secondary resistance to treatment which has led to the development of a new era of second generation tyrosine kinase inhibitors of which nilotinib (Tasigna ${ }^{\circledR}, \mathrm{N}-[3-[3-(1 \mathrm{H}-$ imadazolyl)propoxy] phenyl]-4-methyl-3-[[4-(3-pyridinyl)2-pyrimidinyl]amino]benzamide; Novartis Pharmaceuticals) is highly effective, based on its balance of potent activity and excellent tolerance profile. The selection of a second generation generation tyrosine kinase inhibitor to rescue patients with imatinib failure will be based on several factors including age, co-morbid medical problems and ABL kinase mutational profile. It should be noted that while the use of targeted BCR-ABL kinase inhibitors in CML represents a paradigm shift in CML management these agents are not likely to have activity against the quiescent CML stem cell pool which lead to disease progression or relapse in some patients.

\section{Rationale for second-generation tyrosine kinase inhibitors}

The pivotal IRIS trial (IM Compared with Interferon and Low Dose Cytarabine for Newly Diagnosed Chronic Phase Chronic Myeloid Leukemia), designed to compare prospectively the administration of IM daily to the combination of interferonalfa and cytarabine, recruited 1106 CML-CP patients across 16 countries and was published in the New England Journal of Medicine in 2003. ${ }^{5}$ With a median follow up of 19 months, $96 \%$ of patients achieved a complete hematological response (CHR) on the IM arm, 87\% a major cytogenetic response (MCyR) and $76 \%$ of patients achieved a complete cytogenetic response (CCyR). With 6 years of follow up, ${ }^{6} \mathrm{CHR}$ and $\mathrm{CCyR}$ rates were $97 \%$ and $83 \%$ respectively. An estimated $88 \%$ of patients remained alive and $93 \%$ of patients had not progressed from chronic phase disease. Despite these impressive data, disease progression may occur at an annual rate of $4 \%$ in the first three years of therapy in $\mathrm{CP}$ patients ${ }^{7}$ and in patients with more advanced disease, response rates to IM are poor. The most likely explanation of apparent IM "failure" in most patients is of poor compliance with therapy. An unwelcome consequence of long-term therapy with an agent such as IM which is associated with minimal adverse events is a decrement in patient compliance. A new challenge facing clinicians treating CML is the management of IM resistance (primary or secondary) and/ or intolerance. Primary IM resistance is defined by failure to reach specific hematologic and cytogenetic milestones within the first 18 months of therapy whilst secondary resistance refers to loss of an initial objective response (Table 1).

\section{IM intolerance}

IM therapy is well tolerated in the majority of patients; however, a small group will be intolerant of therapy. Most reactions are of mild-to-moderate grade, but from available data the drug is discontinued for drug-related adverse reactions in $2.4 \%$ of newly diagnosed patients, in $4 \%$ of patients in chronic phase after failure of interferon-alpha therapy, in $4 \%$ of patients with AP and in 5\% with BP. The most frequently reported drug-related adverse reactions are edema, nausea and vomiting, muscle cramps, musculoskeletal pain, diarrhea and rash. The frequency of severe superficial edema is approximately $1.5 \%$ to $6 \%$. These reactions appear to be dose related and are more common in older patients and in those with blast crisis and accelerated phase disease. Severe elevation of transaminases or bilirubin occurs in approximately $5 \%$ of IM-treated CML patients. Treatment was discontinued permanently because of liver laboratory abnormalities in less than $1.0 \%$ of these. With respect to hematologic toxicity, neutropenia and thrombocytopenia are consistent findings. The occurrence of cytopenias in CML patients is also dependent on the stage of the disease. The median duration of the neutropenic and thrombocytopenic episodes varies from 2 to 3 weeks, and from 2 to 4 weeks, respectively. ${ }^{8}$ In general, adverse reactions can usually be managed with either a reduction of the dose or an interruption of treatment along with appropriate supportive measures (eg, growth factor support, diuretics, anti-emetics). In rare cases, however, permanent cessation of therapy is the only solution to manage the intolerant patient.

\section{IM resistance}

Many point mutations within the Abl-kinase protein have been reported - some appear, in some patients, to 
Table I Management of CML - Recommendations from the European LeukemiaNet (ELN): Proposed criteria for failure, suboptimal response, and warnings for previously untreated patients with early chronic phase CML who are treated with IM $400 \mathrm{mg}$ daily

\begin{tabular}{|c|c|c|c|}
\hline Time & Failure & “Suboptimal” response & "Warnings" \\
\hline Diagnosis & N/A & N/A & High risk, del9q+, ACAs in $\mathrm{Ph}+$ cells \\
\hline 3 months after diagnosis & $\begin{array}{l}\text { No HR (stable disease or disease } \\
\text { progression) }\end{array}$ & Less than $\mathrm{CHR}$ & N/A \\
\hline 6 months after diagnosis & Less than $\mathrm{CHR}$, no $\mathrm{CgR}(\mathrm{Ph}+>95 \%)$ & Less than $\mathrm{PCgR}(\mathrm{Ph}+>35 \%)$ & N/A \\
\hline 12 months after diagnosis & Less than $\mathrm{PCgR}(\mathrm{Ph}+>35 \%)$ & Less than $\mathrm{CCgR}$ & Less than MMoIR \\
\hline I8 months after diagnosis & Less than $\mathrm{CCgR}$ & Less than MMolR & $N / A$ \\
\hline Any time & $\begin{array}{l}\text { Loss of } \mathrm{CHR}^{*} \text { Loss of } \mathrm{CCgR}^{\dagger} \\
\text { Mutation }{ }^{\ddagger}\end{array}$ & $\begin{array}{l}\text { ACA in } \mathrm{Ph}+\text { cells }^{\S} \\
\text { Loss of } \mathrm{MMolR}^{\S} \\
\text { Mutation }\end{array}$ & $\begin{array}{l}\text { Any rise in transcript level. Other } \\
\text { chromosomal abnormalities in } \mathrm{Ph}- \\
\text { cells }\end{array}$ \\
\hline
\end{tabular}

*To be confirmed on two occasions unless associated with progression to accelerated phase/blast crisis.

†To be confirmed on two occasions unless associated with CHR loss or progression to accelerated phase/blast crisis.

\#High level of insensitivity to IM.

${ }^{\S} \mathrm{To}$ be confirmed on two occasions unless associated with CHR or CCgR loss.

\#Low level of insensitivity to IM.

Definitions of failure, "suboptimal" response, and "warnings"

- 'Failure' indicates that continuing IM treatment at the current dose is no longer appropriate for the patient.

- 'Suboptimal response' indicates the patient may gain benefit from continuing IM therapy but long-term outcomes may not be favorable.

- 'Warnings' are indications that standard-dose IM treatment may not be the best option for a particular patient, and careful monitoring is required.

Abbreviations: IM, imatinib mesylate; N/A, not applicable; ACA, additional chromosomal abnormalities; HR, hematologic response; CCgR, complete cytogenetic response;

$\mathrm{PCgR}$, partial CgR; CHR, complete hematological response; MCyR, major cytogenetic response; CCyR, complete cytogenetic response; MMolR, major molecular remission.

be mediators of IM resistance. ${ }^{9}$ IM binding to Bcr-Abl produces a closed inactive conformation; however, p-loop point mutations can change the crystal structure of Bcr-Abl producing an open configuration such that IM binding is not possible. ${ }^{10,11}$ The T315I mutation consists of a single threonine substitution for an isoleucine residue at position 315 of the Abl-kinase domain and gives rise to a highly resistant kinase present in $50 \%$ to $90 \%$ of relapsed CML patients. ${ }^{12-15}$ Other mutations target the activation loop and the catalytic domain of the molecule. It is very important to note that there is no current role for "screening" for BCR-ABL mutations in patients in ongoing response to IM. Amplification of BCR-ABL may account for resistance to IM in a small percentage of cases and these patients may or may not respond to increased doses of the drug. ${ }^{9,16}$ Activation of the SFKs (Src family of kinases) and over-expression of Lyn kinase was postulated to potentially have a role in IM resistance. ${ }^{17}$ However as the clinical data have shown no clinical advantage to agents with both Bcr-Abl and Src inhibitory activity, eg, dasatinib, over the safer, more targeted nilotinib, which has no Src inhibitory activity, it seems increasingly unlikely that Src has any significant role in clinical IM resistance. The presence of p-glycoprotein efflux pumps through MDR-1 gene expression ${ }^{18}$ and plasma binding of $\alpha-1$ acid glycoprotein ${ }^{19}$ with IM both prevent the drug reaching its target protein and may be responsible for treatment failure in some cases.
Given the new unmet needs in CML that emerged following IRIS, investigators began to explore the development of new compounds to overcome IM resistance (Figure 1).

Based on crystallographic studies, it was suggested that the potency and selectivity of IM could be improved by incorporating alternative binding groups to the $\mathrm{N}$-methylpiperazine moiety of the compound. This led to the development of nilotinib, a high affinity aminopyrimidine-based ATP competitive inhibitor (Figure 2).

\section{Summary of pre-clinical development}

The recommended dose for nilotinib in IM resistant/intolerant all phase CML is $400 \mathrm{mg}$ twice daily by mouth. ${ }^{20}$ Like IM, nilotinib binds to the inactive conformation of the Abltyrosine kinase, causing P-loop folding over the ATP binding site and activation loop blockade of the substrate binding site. These events disrupt ATP/substrate binding and inhibit the catalytic activity of the enzyme. ${ }^{21}$ The structural similarities of both IM and nilotinib result in a similar profile of protein kinase targets. Dasatinib, which was designed originally as a potential immunosuppressive agent, ie, a Src-kinase inhibitor, has a very different structure and many more off-target kinase targets compared to IM and nilotinib, which is presumably responsible for the latter agents' relatively toxic clinical profile (Table 2). 


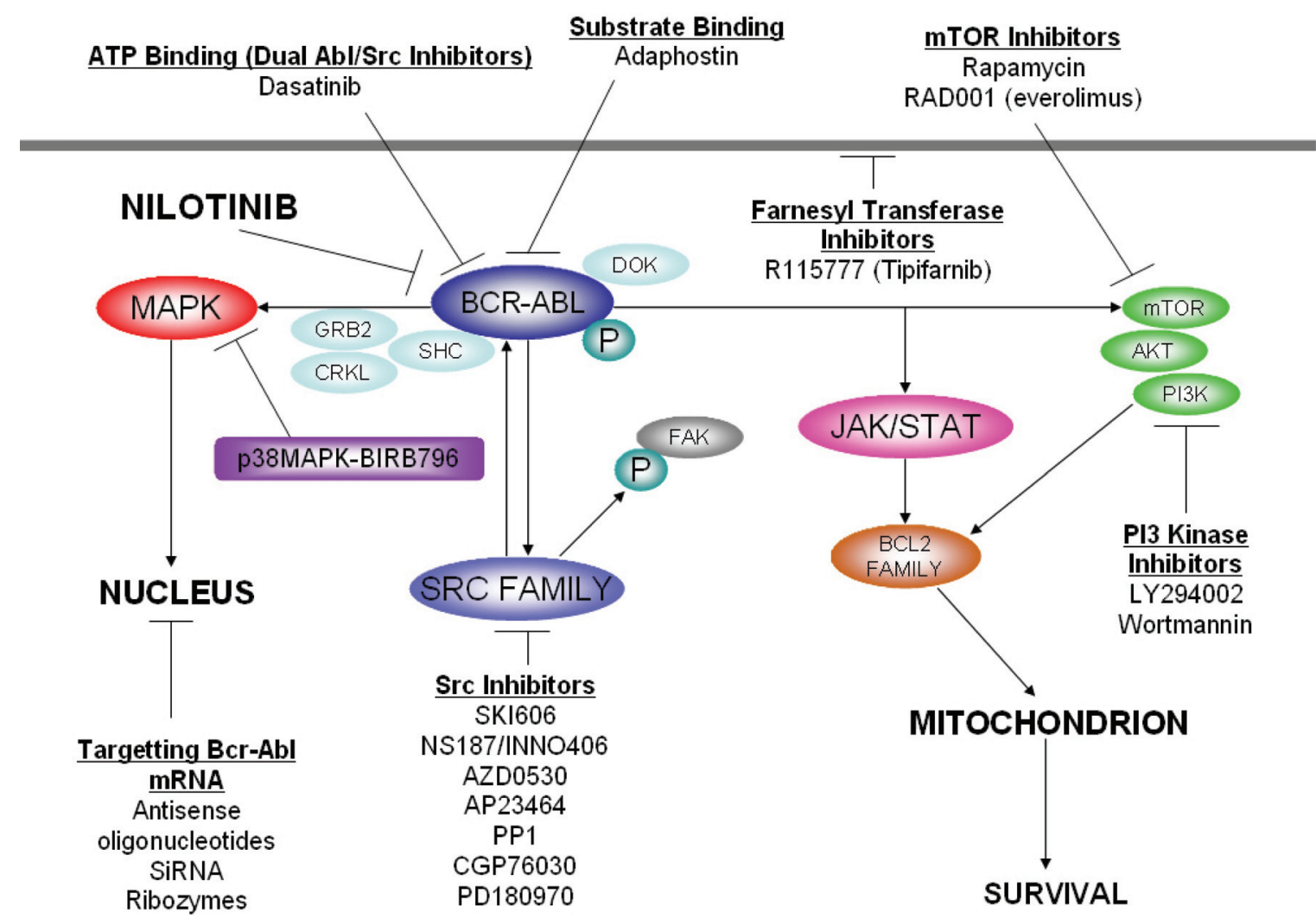

Figure I Targeting the CML signaling cascade..$^{42}$

Abbrevations: ABL, Abelson tyrosine kinase; Akt, Protein kinase B; BCR, breakpoint cluster region; CRKL,V-crk sarcoma virus CTI 0 oncogene homolog (avian)-like; FAK, focal adhesion kinase; FTI, farnesyl transferase inhibitor; Grb-2, growth factor receptor-bound protein 2; JAK, Janus kinase; MAPK, mitogen-activated protein kinase; P, phosphate group; PI3K, phosphatidylinositol-3-kinase; SFK, Src family kinases; SHC, Src homology 2 domain-containing; SRC, homolog of Rous sarcoma virus; Stat, signal transducer and activator of transcription.

In IM-sensitive CML cell lines, nilotinib was 10- to 60-fold more potent an inhibitor than IM. ${ }^{22-25}$ The inhibitory activity of nilotinib is also several fold higher (3- to 7-fold) than that of IM in IM-resistant CML cell lines; however, no activity was seen in cell lines harboring the T315I mutation. ${ }^{24,25}$ Preclinical data indicate that nilotinib effectively inhibits wild type BCR-ABL. At $\mathrm{IC}_{50}$ concentrations of over $150 \mathrm{nM}$, nilotinib was inactive against T315I (Figure 3).

Other protein kinase targets of nilotinib include TEL-PDGFR $\beta$, FIP1L1-PDGFR $\alpha$ and D816V mutated KIT. ${ }^{26-30}$ Nilotinib, therefore, may have a place in the treatment of idiopathic hypereosinophilic syndrome (HES), chronic myelomonocytic leukemia (CMML) and systemic mastocytosis (SM). Kinase domain mutations seem to be less frequent with nilotinib treatment and may respond to higher doses of the drug when they do occur. ${ }^{14}$ Murine models injected with IM-resistant Bcr-Abl cell lines survived longer with nilotinib treatment compared to controls. ${ }^{24,25}$ Other preclinical studies suggest an additive effect when nilotinib is combined with IM. ${ }^{23,31}$ Pharmacokinetic data are available from a phase 1 dose escalation study published by Kantarjian and Giles et al in $2006 .{ }^{32}$ Nilotinib was administered orally at doses of 50 to $1200 \mathrm{mg}$ once daily and at doses of $400 \mathrm{mg}$ or $600 \mathrm{mg}$ twice daily. All trough serum concentrations exceeded the $\mathrm{IC}_{50}$ values for cellular phosphorylation of BCR-ABL including 32 of 33 BCR-ABL mutants. Peak serum concentrations and area under the curve (AUC) values increased with once daily doses of 50 to $400 \mathrm{mg}$ but at higher doses these values began to plateau, likely reflecting saturation of gastrointestinal absorption. At the recommended $400 \mathrm{mg}$ twice daily dosing mean peak and trough serum drug concentrations were respectively $3.6 \mu \mathrm{mol} / \mathrm{L}$ and $1.7 \mu \mathrm{mol} / \mathrm{L}$ at 3 hours. The apparent elimination half-life of nilotinib was close to 17 hours, more than $90 \%$ of an administered dose is eliminated in the feces within 7 days and $70 \%$ of the dose is accounted for by the parent drug. ${ }^{20} \mathrm{Co}$-administration of nilotinib with ketoconazole in healthy volunteers resulted in a 3-fold increased systemic exposure to nilotinib, indicating that nilotinib metabolism is influenced by cytochrome P450 (CYP) 3A4 inhibition..$^{32}$ In the same analysis, co-administration of nilotinib with midazolam resulted in a $30 \%$ increase in midazolam exposure, implying that nilotinib 
may be a weak inhibitor of CYP3A4. Therefore, concomitant use of both CYP3A4 inhibitors (eg, azole antifungals, clarithromycin, atazanavir, ritonavir) and CYP3A4 inducers (eg, dexamethasone, phenytoin, carbamazapine) should be avoided. Based on embryo-fetal developmental studies in pregnant rats and rabbits, nilotinib is not considered to be teratogenic; however, there are no well controlled studies with nilotinib in pregnant women taking the drug. A pharmacogenetic analysis of 97 patients evaluated polymorphisms of UGT1A1 and the potential for hepatic impairment to occur (hyperbilirubinemia) in this group. The (TA)7/(TA)7 genotype was associated with a statistically significant increase in the risk of hyperbilirubinemia relative to the (TA)6/(TA) 6 and (TA)6/(TA) 7 genotypes. $^{20}$

\section{Summary of clinical development}

The therapeutic efficacy of nilotinib has been extensively evaluated in CML. The phase I component of the study consisted of a dose escalation study assessing the safety and tolerability of nilotinib in IM-resistant CML and Ph+ ALL, nilotinib efficacy was assessed a secondary endpoint. The larger phase II trial recruited across numerous international leukemia centers of excellence. The protocol included 6 arms; 3 of those arms involved patients with IM failure in chronic phase, accelerated phase and blastic phase CML, ${ }^{33-35} 2$ arms considered all phase CML patients with prior IM and dasatinib failure, and the remaining arm included patients with $\mathrm{Ph}+\mathrm{ALL}$ and rarer disorders such as the hyper-eosinophilic syndrome and systemic mastocytosis. To date, the results of the phase I study of both the phase II CP and AP CML trials have been published in mature form while initial data on the other studies are available. All of the trials included patients who had previously received IM therapy but were either unable to tolerate the drug or had either primary or secondary resistant disease. Throughout the conducted studies, definitions of remission and response are based on those put forward by the European Leukemia Network for CML (Table 3).

\section{Safety and tolerability}

The first study in man of nilotinib was a multicenter, dose escalation study of orally administered drug on a continuous daily dosing schedule in adults with IM-resistant/intolerant all phase $\mathrm{Ph}+\mathrm{CML}$ and $\mathrm{Ph}+\mathrm{ALL}{ }^{32}$ The primary objectives of the trial were to determine the safety and tolerability of nilotinib and to characterize its biologic and pharmacokinetic profiles. Hematologic and cytogenetic responses were evaluated as

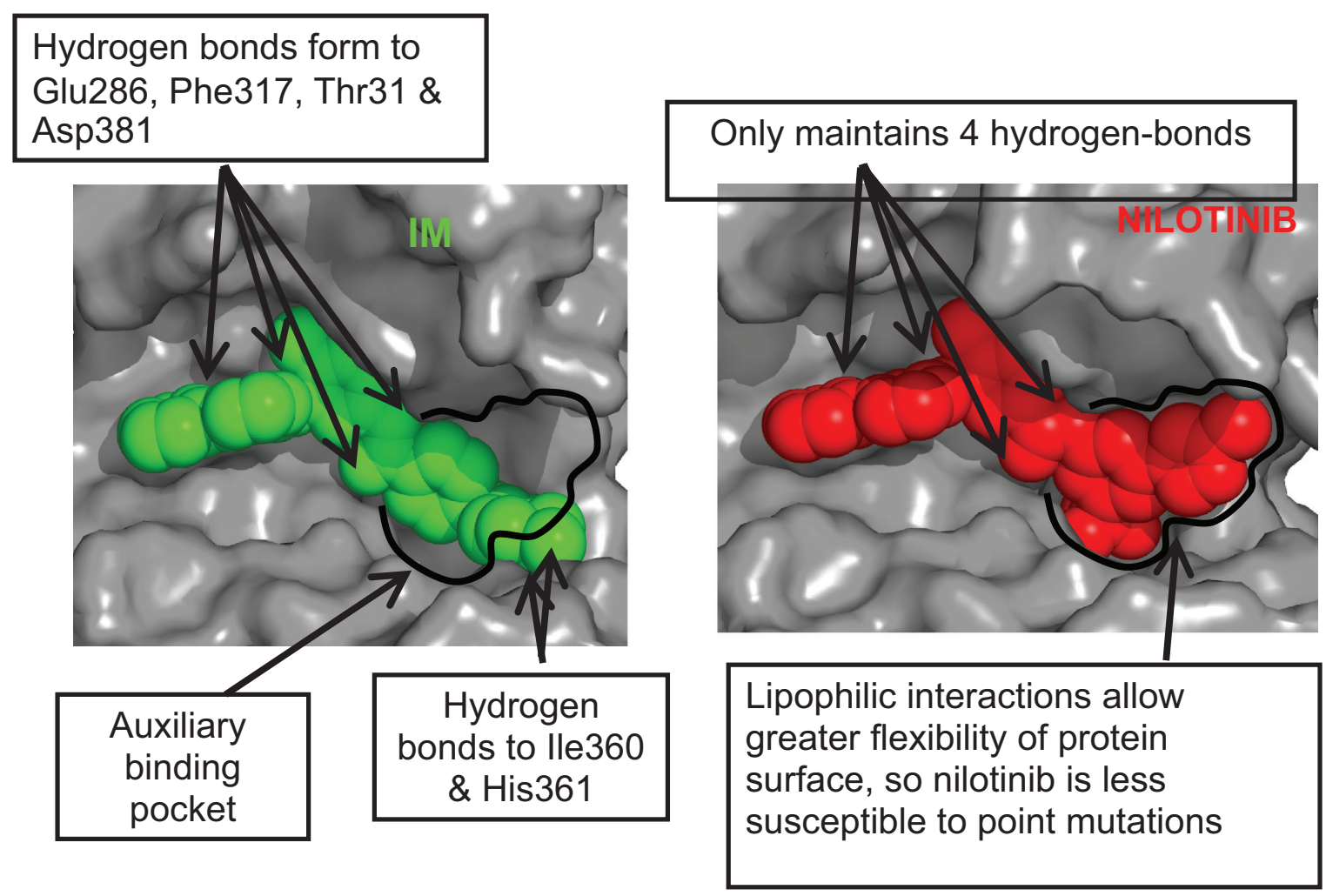

Figure 2 Interaction of imatinib and nilotinib with BCR-ABL.The reduced requirement for hydrogen bonding with nilotinib compared to imatinib, allows for a tighter steric fit into the ATP binding pocket. 
Table 2 Targets of the first- and second-generation tyrosine kinase inhibitors

\begin{tabular}{lllll}
\hline Imatinib & Nilotinib & Dasatinib & & \\
\hline ABL & ABL & ABL & DDRI & MYTI \\
ARG & ARG & ARG & DDR2 & NLK \\
BCR-ABL & BCR-ABL & BCR-ABL & ACK & QIK \\
KIT & KIT & KIT & ACTR2B \\
PDGFR & PDGFR & PDGFR & ACVR2 & QSK \\
DDRI & DDRI & SRC & BRAF & RAFI \\
NQO2 & NQO2 & YES & EGFR/ERBBI & RIPK2 \\
& & FYN & EPHA2 & SLK \\
& & LYN & EPHA3 & STK36/ULK \\
& & HCK & EPHA4 & SYK \\
& & LCK & EPHA5 & TA03 \\
& & FGR & FAK & TESK2 \\
& & GLK & GAK & ZAK \\
& & FRK & GCK & HH498/TNNI3K \\
& & CSK & ILK & LIMKI \\
& BTK & LIMK2 & \\
& TEC & BMX & TXK & \\
& & & & \\
& & & &
\end{tabular}

Notes: The structural similarities of both imatinib and nilotinib result in a similar very limited profile of protein kinase targets. Dasatinib, developed originally as a Src-kinase inhibitor, has a much broader range of non Bcr-Abl targets.

secondary endpoints. The trial recruited 119 patients (106 with $\mathrm{Ph}+\mathrm{CML}$ and 13 with $\mathrm{Ph}+\mathrm{ALL}$ ) who were assigned to get either a 50 to $1200 \mathrm{mg}(\mathrm{n}=69)$ dose once daily or a $400 \mathrm{mg}$ or $600 \mathrm{mg}$ dose twice daily $(\mathrm{n}=40)$. The protocol was amended to allow for dose escalation in patients with an inadequate initial response to therapy in the absence of significant side effects. The median duration of therapy ranged from 4.9 to 5.1 months among patients with CP or AP disease, and was shorter in patients with myeloid (2.9 months) versus lymphoid (1.4 months) BP. The most common adverse events reported were mild to moderate skin rashes, transient and clinically insignificant hyperbilirubinemia and myelosuppression. Grade 3/4 neutropenia occurred in $9 \%$ of patients who got nilotinib at the $400 \mathrm{mg}$ twice daily dose and in $22 \%$ of patients that received the $600 \mathrm{mg}$ twice a day dose (Table 4).

In the larger phase II trial of nilotinib in IM-resistant/ intolerant CP-CML, 280 patients were evaluable for safety and tolerability. ${ }^{33}$ The most frequent grade 3 or 4 hematologic toxicities reported in this study were neutropenia (29\%) and thrombocytopenia ( $29 \%$ also); the median duration for both events was 15 and 22 days respectively.

Dose interruptions were required in $10 \%$ and $19 \%$ of patients and platelet transfusions or growth factor support was required in $10 \%$ and $5 \%$ of patients respectively. Of the non-hematologic toxicities observed, the most common were rash, nausea, pruritus, headache and fatigue. Grade 3 or 4 elevation in AST or ALT levels were infrequent ( $1 \%$ and $4 \%$ of patients respectively), grade $3 / 4$ hyperbilirubinemia occurred in $9 \%$ of cases (likely related to the UGT1A1 polymorphism discussed previously), $14 \%$ of patients had elevated lipase levels and pancreatitis was seen in $1 \%$ of patients. Prolongation of the QT interval (by over $500 \mathrm{msec}$ ) from baseline was seen in $1 \%$ of cases. Preclinical signal in assays of potential to prolong QTc has given very similar data on nilotinib and dasatinib. Nilotinib has been studied in normal volunteers and has no pro-arrythmogenic potential - this study has not yet been performed with dasatinib. Fluid retention events (weight gain, edema, pleural and pericardial effusions) occurring in trials with $\mathrm{IM}^{36}$ and dasatinib were not commonly seen in patients treated with nilotinib. Only 3 grade $1 / 2$ events occurred in the phase II CML-CP study ${ }^{33}$ and none were reported in the phase II CML-AP trial (Table 6). The toxicity data for blastic phase CML in patients treated with dasatinib as second-line therapy are presented in Table 5 for comparison. ${ }^{37}$

In the second published phase II trial of nilotinib in patients with IM-resistant or -intolerant AP reported on by le Coutre et al, ${ }^{34} 119$ patients were enrolled and all were evaluable for safety and tolerability. Similar to the CML-CP 


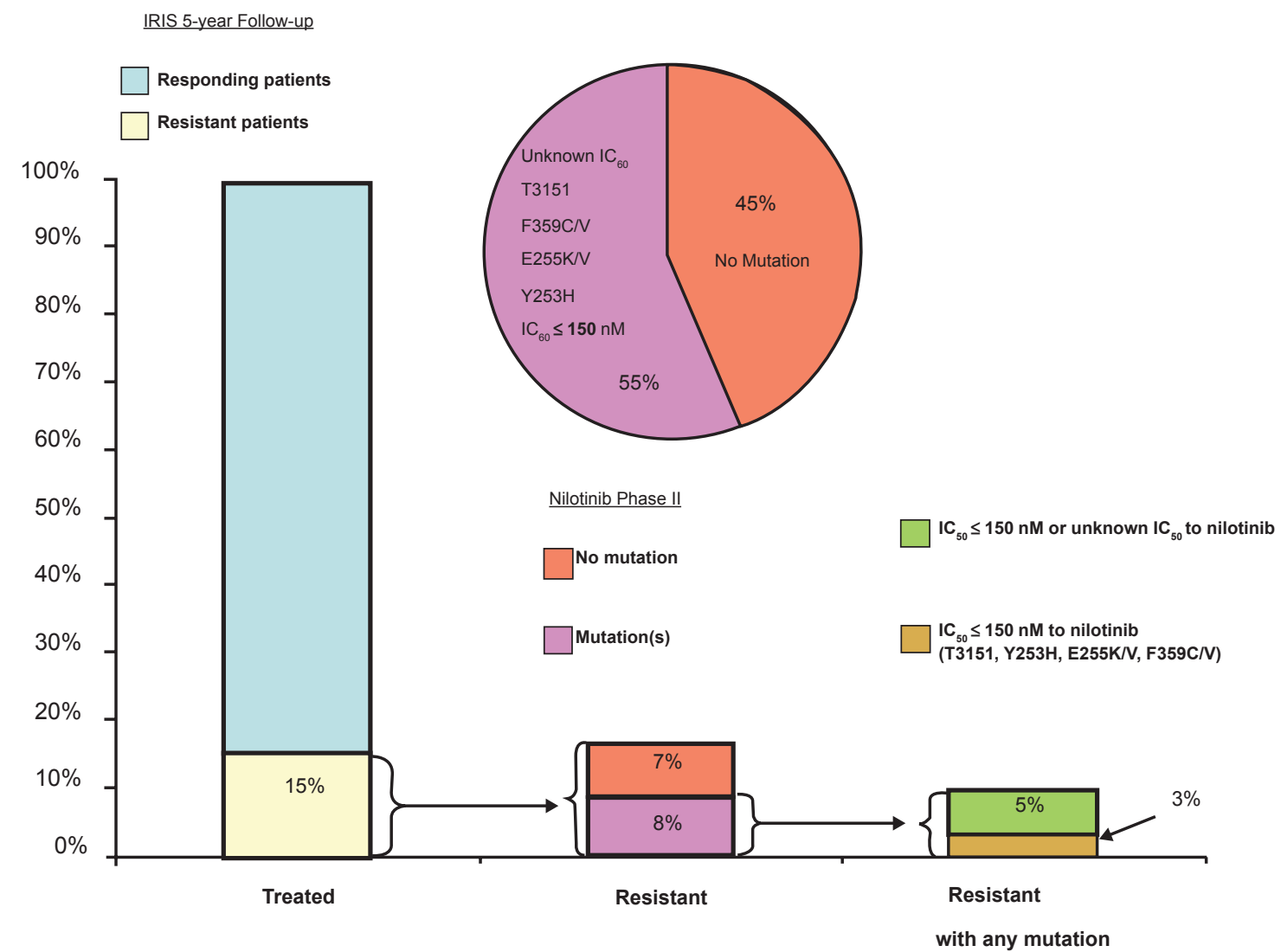

Figure 3 Distribution of mutated phenotypes in imatinib-resistant or -intolerant CML.

study ${ }^{33}$ rash (22\% of cases), pruritus (20\%), constipation $(11 \%)$, headache $(10 \%)$, fatigue $(10 \%)$ and nausea $(10 \%)$ were the most frequent non-hematologic events. Grade 3 or 4 adverse events were uncommon. The most common grade $3 / 4$ hematologic toxicities were anemia, neutropenia and thrombocytopenia in $13 \%, 21 \%$ and $35 \%$ of patients respectively. No clinically significant electrocardiographic changes were observed in this trial. Safety and tolerability data from the other phase II trial of CML-BP patients is broadly comparable to the chronic and accelerated phase II studies.

Data from the ENACT study (Expanding Nilotinib Access in Clinical Trials) was presented at the European Hematology Association (EHA) in June 2008..$^{38}$ This ongoing open label, multicenter trial was initiated to provide expanded access to nilotinib and to obtain additional safety information

Table 3 Most published reports use the same definitions of hematologic and cytogenetic responses, with minor variations (the definitions proposed by ELN are summarized here)

\begin{tabular}{|c|c|c|}
\hline & Definition & Monitoring \\
\hline Hematologic response (complete) & $\begin{array}{l}\text { Platelet count }<450 \times 109 / \mathrm{L} \text { WBC } \\
\text { count }<10 \times 109 / \mathrm{L} . \text { Differential: without } \\
\text { immature granulocytes and with }<5 \% \\
\text { basophils. Non-palpable spleen }\end{array}$ & $\begin{array}{l}\text { Check every } 2 \text { weeks until complete response } \\
\text { achieved and confirmed, then every } 3 \text { months } \\
\text { unless otherwise specified }\end{array}$ \\
\hline Cytogenetic response & $\begin{array}{l}\text { Complete: Ph+ none. Partial: } \\
\text { Ph+ I\%-35\%. Minor: } \mathrm{Ph}+36 \%-65 \% \\
\text { Minimal: } \mathrm{Ph}+66 \%-95 \% . \text { None: } \mathrm{Ph}+>95 \%\end{array}$ & $\begin{array}{l}\text { Check every } 6 \text { months until complete response } \\
\text { achieved and confirmed }\end{array}$ \\
\hline $\begin{array}{l}\text { Molecular response (BCR-ABL: } \\
\text { control gene ratio according to } \\
\text { an international scale) }\end{array}$ & $\begin{array}{l}\text { Complete: transcript non-detectable } \\
\text { Major: } \leq 0.1 \%\end{array}$ & $\begin{array}{l}\text { Check every } 3 \text { months; mutational analysis } \\
\text { only in case of failure, suboptimal response } \\
\text { or increased level of transcript }\end{array}$ \\
\hline
\end{tabular}

Abbrevation: WBC, white blod cells. 
Table 4 Toxicity data from the phase I safety tolerability trial

\begin{tabular}{|c|c|c|}
\hline \multirow[t]{2}{*}{ Adverse event } & \multicolumn{2}{|c|}{ Toxicity grade $(n=119)$} \\
\hline & Grade I or 2 (\%) & Grade 3 or 4 (\%) \\
\hline \multicolumn{3}{|l|}{ Non-hematological event } \\
\hline Rash (all types) & 20 & 2 \\
\hline Pruritus & 15 & 2 \\
\hline Dry skin & 12 & 0 \\
\hline Constipation & 8 & 0 \\
\hline Nausea, vomiting or both & 8 & 0 \\
\hline Increase in both total and conjugated bilirubin levels & 5 & 3 \\
\hline Fatigue & 5 & I \\
\hline Increase in unconjugated bilirubin level & 2 & 4 \\
\hline Alopecia & 6 & 0 \\
\hline Increase in lipase level & 0 & 5 \\
\hline Increase in level of ALT, AST, or both & I & 3 \\
\hline \multicolumn{3}{|l|}{ Hematological event } \\
\hline Thrombocytopenia & I & 20 \\
\hline Neutropenia & I & 13 \\
\hline Anemia & 3 & 6 \\
\hline
\end{tabular}

Notes: Grade 3 or 4 events were primarily hematological as expected. Most adverse events were mild and non-hematological. Grade $3 / 4$ hyperbillirubinemia was rare and primarily indirect and related to polymorphisms in the UCTIAI gene. The toxicity profiles of the subsequent all phase CML studies were comparable to the phase I study.

in patients with all phase CML with prior IM resistance/ intolerance. Patients received nilotinib at the $400 \mathrm{mg}$ twice daily dose. Dose escalation was not permitted. Patients that required a dose reduction to $400 \mathrm{mg}$ once a day were allowed to have their doses escalated to twice daily after resolution of toxicity, lack of response or persistent disease at the discretion of the investigator. More than 1600 patients have been enrolled and results were available for 1056 patients $(\mathrm{CP}, \mathrm{n}=818, \mathrm{AP}, \mathrm{n}=120, \mathrm{BP}, \mathrm{n}=118)$. The main toxicities reported for nilotinib therapy were similar to those in the phaseI/II studies discussed above. The majority of adverse events were hematologic, with the most common grade $3 / 4$ toxicities being thrombocytopenia (22\%) and neutropenia (13\%). Non-hematologic toxicities were mostly mild and included headache, rash and nausea. Death occurred in 28 (3\%) of patients and was more common in those with BP disease $(n=15)$. An average sudden death rate of $0.6 \%$ annually has been observed among patients on the nilotinib studies which is directly comparable to that seen on other studies in patients with CML receiving IM or dasatinib therapy. Across the large phase II trials of nilotinib, the median doses received were very close to the planned treatment doses of $800 \mathrm{mg}$ daily (Figure 4).

Cross intolerance between IM and nilotinib is negligible, reflecting the more effective targeting of Bcr-Abl by the latter agent and thus nilotinib is the agent of choice in patients with all forms of IM intolerance (Figure 2) ${ }^{39}$

\section{Efficacy data}

Efficacy data are available from the phase I trial ${ }^{32}$ and the three phase II studies ${ }^{33-35}$ conducted in patients with CP, AP and BP CML as well as a nilotinib third-line phase II trial in patients with both IM and dasatinib failure.

\section{Phase I trial}

In patients with CML-CP, ${ }^{32} 11$ of 17 patients (65\%) achieved a CHR. A major cytogenetic remission occurred in 6 patients (35\%) and 9 patients (53\%) got at least some form of cytogenetic remission (minimal, major or minor). In the patients with CML-AP $(\mathrm{n}=56), 26(46 \%)$ got a CHR and 15 (27\%) achieved a MCyR, 38 patients (74\%) got hematologic remissions (complete response, marrow response, or return to chronic phase disease) and 31 (55\%) achieved cytogenetic remissions. Of the 33 patients with CML-BP, 2 (6\%) had a CHR and $6(18 \%)$ had a MCyR with nilotinib. Thirteen patients $(39 \%)$ got some form of hematologic response and 9 patients (27\%) achieved a cytogenetic response (minimal, major or minor). Patients who failed to respond to initial therapy with nilotinib responded with increased dosing. Of the 23 patients with AP or BP 
Table 5 Toxicity data for dasatinib phase II study in patients with blastic phase $\mathrm{CML}^{37}$

\begin{tabular}{|c|c|c|c|c|}
\hline \multirow[t]{3}{*}{ Adverse event } & \multicolumn{4}{|c|}{ Patients N (\%) } \\
\hline & \multicolumn{2}{|c|}{ MBP-CML $(n=109)$} & \multicolumn{2}{|c|}{ LBP-CML $(n=48)$} \\
\hline & All grades & Grade 3/4 & All grades & Grade $3 / 4$ \\
\hline \multicolumn{5}{|c|}{ Non-hematologic events } \\
\hline Diarrhea & $43(39)$ & $8(7)$ & $16(33)$ & I (2) \\
\hline Pleural effusion & $39(36)$ & $16(15)$ & $6(13)$ & $3(6)$ \\
\hline Vomiting & $24(22)$ & $3(3)$ & $12(25)$ & I (2) \\
\hline Dyspnea & $23(2 \mathrm{I})$ & $7(6)$ & $6(13)$ & I (2) \\
\hline Pyrexia & $22(20)$ & $5(5)$ & $8(17)$ & I (2) \\
\hline Nausea & $21(19)$ & $4(4)$ & $12(25)$ & 0 \\
\hline Fatigue & $20(18)$ & $2(2)$ & $13(27)$ & $2(4)$ \\
\hline Peripheral edema & $20(18)$ & 0 & $6(13)$ & 0 \\
\hline Rash & $15(14)$ & 0 & $8(17)$ & $2(4)$ \\
\hline Febrile neutropenia & $5(5)$ & $5(5)$ & $8(17)$ & $7(15)$ \\
\hline Asthenia & $13(12)$ & $2(2)$ & $4(8)$ & I (2) \\
\hline Anorexia & $12(\mid \mathrm{I})$ & $\mathrm{I}(\mathrm{I})$ & $3(6)$ & 0 \\
\hline Gl hemorrhage & II (I0) & $6(6)$ & 0 & 0 \\
\hline Headache & II (I0) & $2(2)$ & $8(17)$ & I (2) \\
\hline Epistaxis & II (I0) & $2(2)$ & I (2) & 0 \\
\hline Cough & II (I0) & I (I) & $3(6)$ & 0 \\
\hline \multicolumn{5}{|l|}{ Hematological events } \\
\hline Leukocytopenia & & $67(6 \mathrm{I})$ & & $34(7 \mathrm{I})$ \\
\hline Neutropenia & & $87(80)$ & & $39(8 \mathrm{I})$ \\
\hline Thrombocytopenia & & $89(82)$ & & $42(88)$ \\
\hline Anemia & & $75(69)$ & & $24(50)$ \\
\hline
\end{tabular}

Abbreviations: MBP, myeloid blast phase; LBP, lymphoid blast phase.

disease who had a dose escalation from 50 to $400 \mathrm{mg}$ once daily to $400 \mathrm{mg}$ or $600 \mathrm{mg}$ twice daily, 13 (57\%) of those had hematologic responses. Nilotinib was equally effective in those with and without ABL kinase mutations in those that had mutational analysis carried out $(n=91)$.

\section{Phase II trial in chronic phase CML}

In this study, $280 \mathrm{CML}-\mathrm{CP}$ phase patients were evaluated. ${ }^{33}$ The primary end-point of the trial was major cytogenetic response. The median duration of CML was 57 months.
Patients were enrolled with either IM-refractory $(69 \%)$ or -intolerant disease (31\%) and the majority had received prior therapy with interferon (66\%), hydoxyurea (83\%) and/or cytarabine $(25 \%)$. Responses were comparable in both the IM-resistant or -intolerant groups. Major cytogenetic responses were observed in $48 \%$ of patients and CCyRs were seen in $31 \%$ of cases. The median time to MCyR was 2.8 months giving a projected survival at 1 year of $95 \%$. Just 5 patients (4\%) who got a MCyR stopped therapy due to death or disease progression. Complete hematologic

Table 6 Fluid retention events for nilotinib in IM refractory/intolerant CML patients in accelerated phase (AP) and blastic phase (BP) disease

\begin{tabular}{|c|c|c|c|c|}
\hline & \multicolumn{2}{|l|}{$\operatorname{AP}(\mathbf{N}=138)$} & \multicolumn{2}{|l|}{$B P(N=136)$} \\
\hline & All Grades n (\%) & Grades 3 or $4 n$ (\%) & All Grades n (\%) & Grades 3 or 4 n (\%) \\
\hline Peripheral edema & $6(4.4)$ & 0 & $12(9)$ & $\mathrm{I}(<\mathrm{I})$ \\
\hline Pericardial effusion & 0 & 0 & $0(0)$ & $0(0)$ \\
\hline Pleural effusion & 0 & 0 & $(<\mathrm{I})$ & $0(0)$ \\
\hline Pulmonary edema & $\mathrm{I}(0.7)$ & I (0.7) & $0(0)$ & $0(0)$ \\
\hline
\end{tabular}




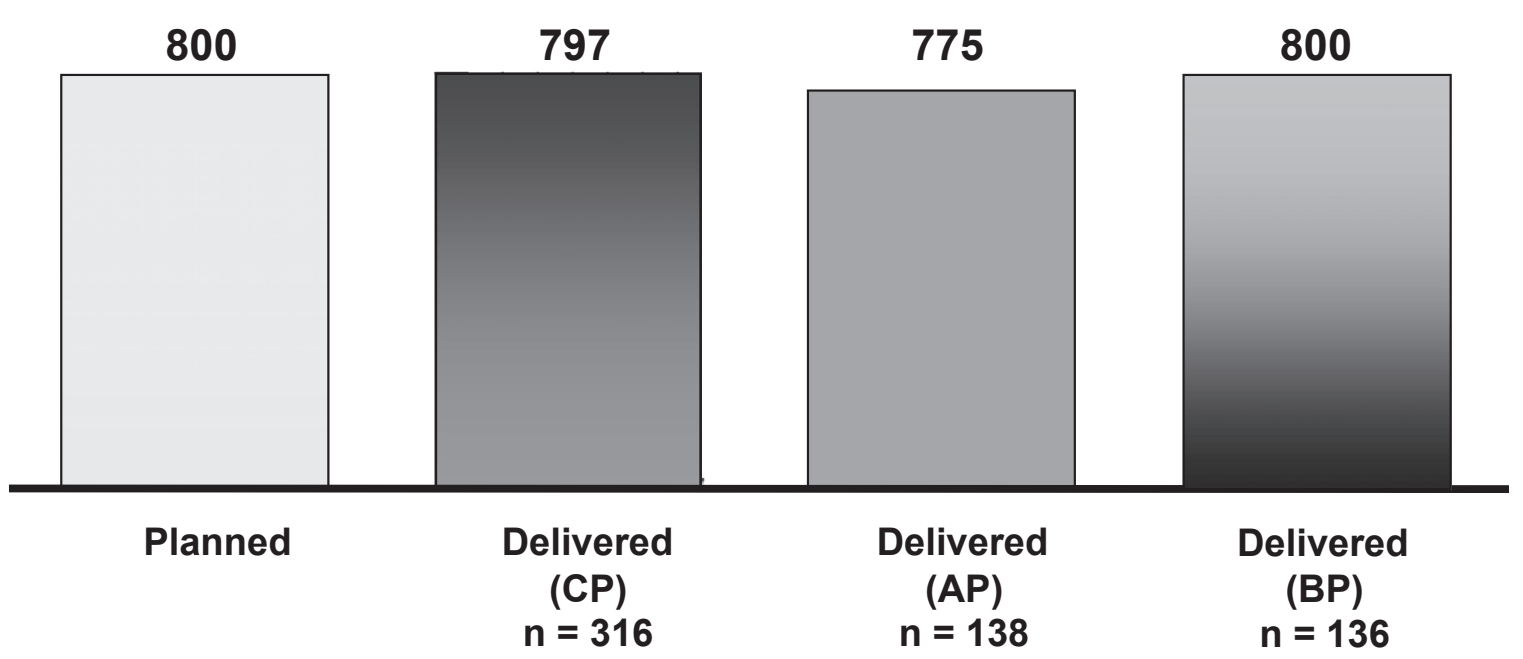

Figure 4 Nilotinib phase II dosing. The planned nilotinib phase II dose was $800 \mathrm{mg}$ per day. The median doses for the chronic phase (CP), accelerated phase (AP) and blastic phase (BP) studies are shown and indicate that nilotinib was well tolerated in all three groups.

responses were seen in 137 of 185 patients who had active disease at enrollment (74\%) and the median time to response in this group was 1 month. Of the patients with ABL kinase mutations, $42 \%$ had a MCyR, compared to $51 \%$ of those without mutations. Results for CCyRs were respectively $23 \%$ and 35\%. Major cytogenetic responses were observed across all BCR-ABL genotypes except in the 4 patients $(2.2 \%)$ that harbored a T315I mutation.

\section{Phase II trial in accelerated phase CML}

In the trial by le Coutre et $\mathrm{al}^{34} 119$ patients with IM-resistant (81\%) or -intolerant (19\%) accelerated phase CML were enrolled. The primary endpoint in this study was hematologic response. Cytogenetic response was an important secondary endpoint. Patients had received a diagnosis of CML for a median duration of 71 months and were on IM therapy for a median of 32 months prior to enrollment. Similar to the phase II CML-CP study, most patients had received treatment with either hydroxyurea, interferon or cytarabine in addition to IM. Patients were treated with $400 \mathrm{mg}$ of nilotinib twice daily; this dose could be increased to $600 \mathrm{mg}$ twice daily as required. The overall hematologic response rate was $47 \%$ with a median time to response of I month. Twenty-six percent of patients achieved a CHR. In the patients who achieved cytogenetic responses, 29\% got a MCyR and $16 \%$ got a CCyR. The median time to major cytogenetic response was 2 months, the median duration of response lasting 15.4 months. At 12 months, the estimated overall survival was $79 \%$ of the 119 patients evaluated. Results of this trial $(n=136)$ were updated at EHA in June 2008.

\section{Phase II trial in blastic phase CML}

Efficacy data for 136 CML-BP patients was presented recently at $\mathrm{EHA}^{35}$ Of these $82 \%$ were IM resistant and $18 \%$ were IM intolerant. The primary endpoint for this trial was confirmed hematologic response. The majority of patients had received prior therapies (34\% had interferon, $37 \%$ had cytarabine and $13 \%$ had been transplanted). At the time of enrollment, $38 \%$ of patients had over $95 \%$ $\mathrm{Ph}+$ metaphases, with additional chromosomal abnormalities in $53 \%$ of patients. Confirmed hematologic response was seen in 29 patients (21\%), and 15 patients (11\%) got a CHR. Major cytogenetic remission was achieved in 55 patients (40\%), and 40 patients (29\%) got CCyRs. Overall survival at 12 months was $42 \%$. Patients with myeloid and lymphoid blast crisis had similar survival outcomes.

\section{Phase II trials of nilotinib as third-line therapy}

Data are available for 42 patients with all-phase CML who failed both IM and dasatinib. ${ }^{40,41}$ Of these, 16 had chronic phase disease, 9 had accelerated phase disease and 17 were in blast crisis. All patients got nilotinib in the standard doses (400 mg BID which could be increased to $600 \mathrm{mg}$ BID if necessary). Median age was 58 (19-78 years) years and the median time elapsed since first diagnosed was 81 (4-420) days. The primary endpoint for CP patients was CyR and the primary endpoint for AP patients was HR. Of the 16 evaluable CP-CML patients, 13 had no baseline CHR. Four of 13 patients (31\%) had a MCyR, 2 complete $(15 \%)$ and 2 partial (15\%). Five of 13 patients (38\%) got a 
Table 7 Phase I/II response rates for nilotinib in three second-line phase II trials and one third-line phase II trial (soon to be published)

\begin{tabular}{|c|c|c|c|c|c|c|c|}
\hline \multirow[t]{2}{*}{ Response } & \multirow{2}{*}{$\begin{array}{l}\text { Phase I study } \\
(\mathrm{n}=119)\end{array}$} & \multicolumn{3}{|c|}{ Phase II second-line studies } & \multicolumn{3}{|c|}{ Phase II third-line study } \\
\hline & & $\begin{array}{l}\text { Chronic phase } \\
(n=280)\end{array}$ & $\begin{array}{l}\text { Accelerated } \\
\text { phase }(n=119)\end{array}$ & $\begin{array}{l}\text { Blastic phase } \\
(n=136)\end{array}$ & $\begin{array}{l}\text { Chronic phase } \\
(n=16)\end{array}$ & $\begin{array}{l}\text { Accelerated } \\
\text { phase }(n=\text { AP })\end{array}$ & $\begin{array}{l}\text { Blastic phase } \\
(n=17)\end{array}$ \\
\hline $\begin{array}{l}\text { Hematological } \\
\text { responses (total) }\end{array}$ & $58 \%$ & NA & $47 \%$ & $21 \%$ & NA & $22 \%$ & NA \\
\hline $\mathrm{CHR}$ & $37 \%$ & $74 \%$ & $26 \%$ & $15 \%$ & $38 \%$ & NA & $18 \%$ \\
\hline $\begin{array}{l}\text { Cytogenetic } \\
\text { responses (total) }\end{array}$ & $55 \%$ & $70 \%$ & $66 \%$ & NA & NA & NA & NA \\
\hline MCyR & $25 \%$ & $48 \%$ & $29 \%$ & $40 \%$ & $31 \%$ & NA & NA \\
\hline CCyR & $15 \%$ & $31 \%$ & $16 \%$ & $29 \%$ & $15 \%$ & NA & NA \\
\hline
\end{tabular}

Abbreviations: CHR, complete hematologic response, MCyR, major cytogenetic response, CCyR, complete cytogenetic response, NA, not available.

CHR. Of the 9 AP patients, 2 (22\%) returned to $\mathrm{CP}, 6(67 \%)$ were not evaluable and there was 1 death (11\%). Of the 17 CML-BP patients $3(18 \%)$ had a CHR, I returned to CP $(6 \%)$, 5 had stable disease (29\%), 4 (24\%) were not evaluable and $4(24 \%)$ had progressive disease (Table 7 and Figure 5).

\section{Conclusions and future directions}

Nilotinib has proven to be optimal second line therapy in CML based on 1) its efficacy in all disease phases; 2) its minimal cross intolerance with IM; 3) its activity across the entire spectrum of BCR-ABL mutations except T315I; 4) its low incidence of grade 3/4 extramedullary AEs (adverse events) which tend to occur early in therapy; 5) its relatively low incidence of severe myelosuppressive AEs; and 6) its considerably superior tolerability over long periods at full prescribed approved doses relative to dasatinib. Phase III studies are underway in patients with newly diagnosed CML, where the goal of treatment will be to prevent the emergence of resistant clones and improve survival over the current standard, IM. Nilotinib has been approved by the FDA for the treatment of adults with CP or AP CML who are either resistant or intolerant of prior therapies including IM. Nilotinib has also been licensed for these indications by the EMEA in Europe and numerous other regulatory agencies. The rapid transition of nilotinib through the regulatory process represents a true triumph of rational anti-cancer agent design and builds on the expectations of the enormous success of IM in the clinic.

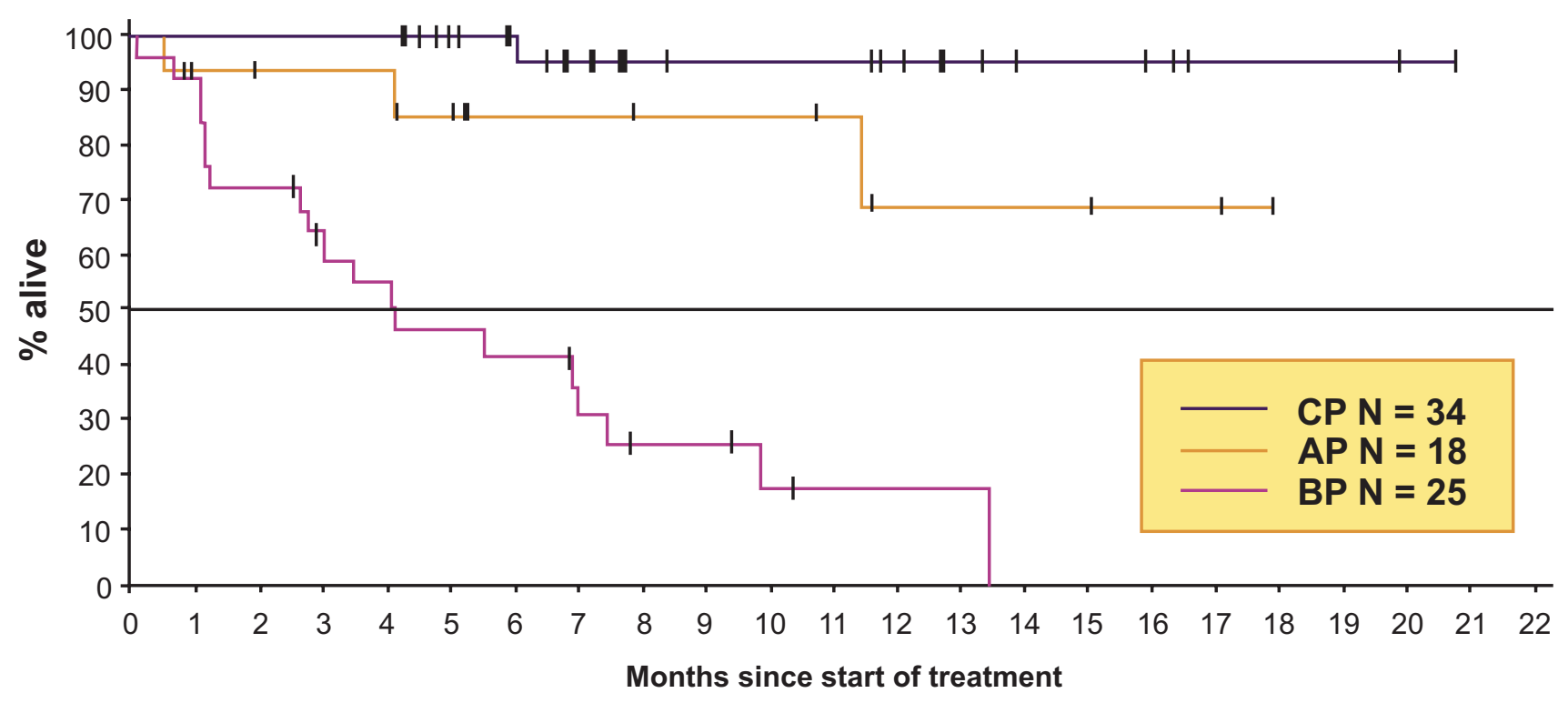

Figure 5 Overall survival for nilotinib as third-line therapy for patients resistant or intolerant of both imantinib and dasatinib. Abbreviations: $\mathrm{CP}$, chronic phase; $\mathrm{AP}$, accelerated phase; $\mathrm{BP}$, blastic phase. 


\section{Disclosures}

None of the authors disclose conflicts of interest.

\section{References}

1. Faderl S, et al. The biology of chronic myeloid leukemia. $N$ Engl J Med. 1999;341(3):164-172.

2. Sawyers CL. Chronic myeloid leukemia. N Engl J Med. 1999;340(17): 1330-1340.

3. Jemal A, et al. Cancer statistics. CA Cancer J Clin. 2006;56(2) 106-130.

4. Druker BJ, et al. Five-year follow-up of patients receiving imatinib for chronic myeloid leukemia. N Engl J Med. 2006;355(23):2408-2417.

5. O'Brien SG, et al. Imatinib compared with interferon and low-dose cytarabine for newly diagnosed chronic-phase chronic myeloid leukemia. N Engl J Med. 2003;348(11):994-1004.

6. Hochhaus A, et al. IRIS 6-year follow-up: sustained survival and declining annual rate of transformation in patients with newly diagnosed chronic myeloid leukemia in chronic Phase (CML-CP) treated with imatinib. Poster presentation at the 2007 American Society of Hematology annual meeting.

7. Silver RT, et al. Four years of follow-up of 1027 patients with late chronic phase (L-CP), accelerated phase (AP), or blast crisis (BC) chronic myeloid leukemia (CML) treated with imatinib in three large phase II trials. Poster presentation at the 2004 American Society of Hematology annual meeting; 2004.

8. Novartis. Online at: http://www.pharma.us.novartis.com/product/pi/ pdf/gleevec_tabs.pdf. 2006.

9. Gorre ME, et al. Clinical resistance to STI-571 cancer therapy caused by BCR-ABL gene mutation or amplification. Science. 2001;293(5531):876-880.

10. Corbin AS, et al. Several Bcr-Abl kinase domain mutants associated with imatinib mesylate resistance remain sensitive to imatinib. Blood. 2003;101(11):4611-4614.

11. Shah NP, et al. Multiple BCR-ABL kinase domain mutations confer polyclonal resistance to the tyrosine kinase inhibitor imatinib (STI571) in chronic phase and blast crisis chronic myeloid leukemia. Cancer Cell. 2002;2(2):117-125.

12. Azam M, et al. Activity of dual SRC-ABL inhibitors highlights the role of BCR/ABL kinase dynamics in drug resistance. Proc Natl Acad Sci US A. 2006;103(24):9244-9249.

13. Griswold IJ, et al. Kinase domain mutants of Bcr-Abl exhibit altered transformation potency, kinase activity, and substrate utilization, irrespective of sensitivity to imatinib. Mol Cell Biol. 2006;26(16):6082-6093.

14. von Bubnoff $\mathrm{N}$, et al. Bcr-Abl resistance screening predicts a limited spectrum of point mutations to be associated with clinical resistance to the $\mathrm{Abl}$ kinase inhibitor nilotinib (AMN107). Blood. 2006;108(4):1328-1333.

15. von Bubnoff $\mathrm{N}$, et al. BCR-ABL gene mutations in relation to clinical resistance of Philadelphia-chromosome-positive leukaemia to STI571: a prospective study. Lancet. 2002;359(9305):487-491.

16. Hochhaus A, La Rosee P. Imatinib therapy in chronic myelogenous leukemia: strategies to avoid and overcome resistance. Leukemia. 2004;18(8):1321-1331.

17. Donato NJ, et al. BCR-ABL independence and LYN kinase overexpression in chronic myelogenous leukemia cells selected for resistance to STI571. Blood. 2003;101(2):690-698.

18. Illmer $\mathrm{T}$, et al. P-glycoprotein-mediated drug efflux is a resistance mechanism of chronic myelogenous leukemia cells to treatment with imatinib mesylate. Leukemia. 2004;18(3):401-408.

19. Gambacorti-Passerini $C$, et al. Role of alphal acid glycoprotein in the in vivo resistance of human BCR-ABL $(+)$ leukemic cells to the abl inhibitor STI571. J Natl Cancer Inst. 2000;92(20):1641-1650.

20. Novartis Pharmaceuticals Corporation: East Hanover (NJ). Tasigna (nilotinib capsules): US prescribing information (online). Available form URL: hhtp//www.fda.gov/.
21. Manley PW, et al. Molecular Interactions between the Highly Selective pan-Bcr-Abl Inhibitor, AMN107, and the Tyrosine Kinase Domain of Abl. Poster presentation at the 2005 American Society of Hematology annual meeting; 2005.

22. Golemovic M, et al. AMN107, a novel aminopyrimidine inhibitor of Bcr-Abl, has in vitro activity against imatinib-resistant chronic myeloid leukemia. Clin Cancer Res. 2005;11(13):4941-4947.

23. O'Hare T, et al. In vitro activity of Bcr-Abl inhibitors AMN107 and BMS-354825 against clinically relevant imatinib-resistant Abl kinase domain mutants. Cancer Res. 2005;65(11):4500-4505.

24. Weisberg E, et al. AMN107 (nilotinib): a novel and selective inhibitor of BCR-ABL. Br J Cancer. 2006;94(12):1765-1769.

25. Weisberg E, et al. Characterization of AMN107, a selective inhibitor of native and mutant Bcr-Abl. Cancer Cell. 2005;7(2):129-141.

26. Gleixner KV, et al. PKC412 inhibits in vitro growth of neoplastic human mast cells expressing the D816V-mutated variant of KIT: comparison with AMN107, imatinib, and cladribine (2CdA) and evaluation of cooperative drug effects. Blood. 2006;107(2):752-759.

27. Prenen $\mathrm{H}$, et al. Cellular uptake of the tyrosine kinase inhibitors imatinib and AMN107 in gastrointestinal stromal tumor cell lines. Pharmacology. 2006;77(1):11-16.

28. Stover EH, et al. The small molecule tyrosine kinase inhibitor AMN107 inhibits TEL-PDGFRbeta and FIP1L1-PDGFRalpha in vitro and in vivo. Blood. 2005;106(9):3206-3213

29. Verstovsek S, et al. Effects of AMN107, a novel aminopyrimidine tyrosine kinase inhibitor, on human mast cells bearing wild-type or mutated codon 816 c-kit. Leuk Res. 2006;30(11):1365-1370.

30. Verstovsek S, et al. Activity of AMN107, a novel aminopyrimidine tyrosine kinase inhibitor, against human FIP1L1-PDGFR-alpha-expressing cells. Leuk Res. 2006;30(12):1499-1505.

31. Bradeen HA, et al. Comparison of imatinib mesylate, dasatinib (BMS-354825), and nilotinib (AMN107) in an N-ethyl-N-nitrosourea (ENU)-based mutagenesis screen: high efficacy of drug combinations. Blood. 2006;108(7):2332-2338.

32. Kantarjian $\mathrm{H}$, et al. Nilotinib in imatinib-resistant CML and Philadelphia chromosome-positive ALL. NEngl J Med. 2006;354(24): 2542-2551.

33. Kantarjian HM, et al. Nilotinib (formerly AMN107), a highly selective BCR-ABL tyrosine kinase inhibitor, is effective in patients with Philadelphia chromosome-positive chronic myelogenous leukemia in chronic phase following imatinib resistance and intolerance. Blood. 2007;110(10):3540-3546

34. le Coutre P, et al. Nilotinib (formerly AMN107), a highly selective BCR-ABL tyrosine kinase inhibitor, is active in patients with imatinibresistant or -intolerant accelerated-phase chronic myelogenous leukemia. Blood. 2008;111(4):1834-1839.

35. Ottman $\mathrm{O}$, et al. Nilotinib monotherapy in patients with imatinibresistant or intolerant $\mathrm{Ph}+$ chronic myeloid leukemia (CML) in blast crisis (BC) or relapsed/refractory $\mathrm{Ph}+$ acute lymphoblastic leukemia (ALL). Poster Presented at the 12th Congress of the European Hematology Association, 2008.

36. Moen MD, et al. Imatinib: a review of its use in chronic myeloid leukaemia. Drugs. 2007;67(2):299-320.

37. Cortes $\mathrm{J}$, et al. Efficacy and safety of dasatinib in imatinib-resistant or -intolerant patients with chronic myeloid leukemia in blast phase. Leukemia. 2008;22(12):2176-2183.

38. Nicolini F, et al. Expanding Nilotinib Access in Clinical Trials (ENACT) Study in Adult Patients with Imatinib-Resistant or Intolerant Chronic Myeloid Leukemia (CML): Updated Safety Analysis. Poster Presentation at the 12th Congress of the European Hematology Association, 2008.

39. Hochaus A, et al. Minimal Cross Intolerance between Nilotinib and Imatinib in Patients with Imatinib-Intolerant Chronic Myeloid Leukemia (CML) in Chronic Phase (CP) or Accelerated Phase (AP). Poster Presentation at the 12th Congress of the European Hematology Association, 2008. 
40. Giles F, et al. Nilotinib therapy after dasatinib failure in patients with imatinib resistant or intolerant chronic phase and accelerated phase Philadelphia chromosome positive chronic myeloid leukemia [abstract no. 0554]. Poster presentation at the 12th Congress of the European Hematology Association (EHA), 2007.

41. Giles F, et al. A phase II study of nilotinib administered to patients with imatinib resistant or intolerant chronic myeloid leukemia (CML) in chronic phase (CP), accelerated phase (AP) or blast crisis (BC) who also failed dasatinib [abstract no. 7038]. Poster presentation at the 43rd Annual Meeting of the American Society of Clinical Oncology. 2007.
42. Swords R, et al. Novel Abl kinase inhibitors in chronic myeloid leukemia in blastic phase and Philadelphia chromosome-positive acute lymphoblastic leukemia. Clin Lymphoma Myeloma. 2007;7 Suppl 3: S113-S119. 
